\title{
Combined Effect of Flubendazole and Praziquantel on Echinococcus granulosus Protoscoleces and Microcysts
}

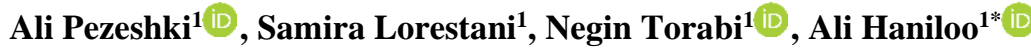 \\ 1. Dept. of Parasitology and Mycology, Faculty of Medicine, Zanjan University of Medical Sciences, Zanjan, Iran
}

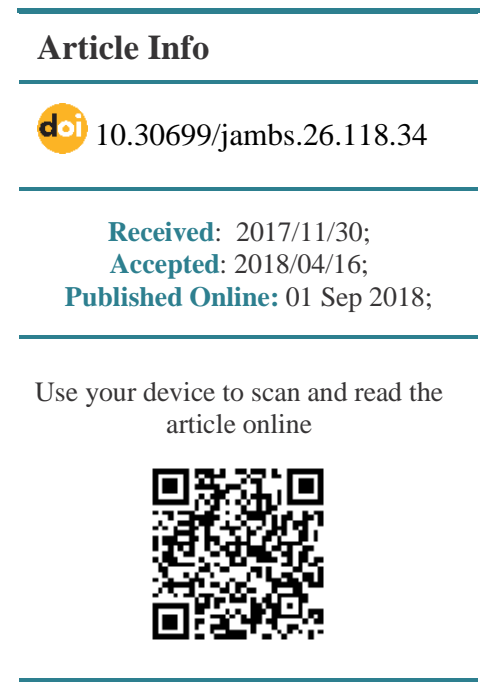

Corresponding Information: Dr. Ali Haniloo,

Dept. of Parasitology and Mycology, Faculty of Medicine, Zanjan University of Medical Sciences, Zanjan, Iran. E-mail: hani@zums.ac.ir

\begin{abstract}
Background \& Objective: Benzimidazoles such as albendazole are being used in some conditions to treat hydatid disease, the common parasitic infections of humans and livestock caused by Echinococcus granulosus. However, treatment with these drugs is not completely successful. In this study we evaluated the in vitro efficacy of flubendazole (FLBZ) and praziquantel (PZQ) against E. granulosus protoscoleces and microcysts.
\end{abstract}

Materials \& Methods: Viable and aseptically cultured protoscoleces were treated for 17 days with $5 \mu \mathrm{g} / \mathrm{mL}$ FLBZ, $1 \mu \mathrm{g} / \mathrm{mL}$ PZQ and a combination of both drugs. The viability of protoscoleces were assessed daily by microscopic observation and eosin test. The experiment was repeated three times and drug efficacies were presented as the mean value of protoscoleces' viability rate. Developed microcysts from the continued cultivation of protoscoleces were treated with the same doses of the drugs for a period of 21 days. The structural changes of the microcysts were visualized by inverted and transmission electron microscopy.

Results: The most protoscolicidal efficacy was detected in the culture treated with the combination of FLBZ and PZQ, so that all protoscoleces were killed in nine days following the beginning of treatment. Meanwhile, $21.7 \pm 9.5 \%$ and $30.8 \pm 5.3 \%$ of protoscoleces that were incubated with the FLBZ or PZQ alone were still viable, respectively. Degenerative changes occurred in microcysts after four days of treatment with both drugs, while these changes occurred six and eight days later with FLBZ or PZQ alone, respectively.

Conclusion: This study indicated the favorable combined effect of FLBZ and PZQ against $E$. granulosus protoscoleces and microcysts in vitro.

Keywords: Echinococcus granulosus, Microcyst, Flubendazole, Praziquantel which permits copy and redistribute of the material just in noncommercial usages with proper citation.

\section{Introduction}

Cystic hydatid disease or cystic echinococcosis (CE) is a parasitic zoonosis caused by the larval stage (metacestode) of the Echinococcus granulosus tapeworm, and it has worldwide distribution. Human $\mathrm{CE}$ is a major health problem in endemic areas including South America, the Mediterranean, the Middle East and central Asia (1). According to WHO reports, in areas where the disease is endemic, incidence rates of human CE can exceed 50 per 100 000 person-years (2). Adult E. granulosus survives in the small intestine of canids (final host) and the embryonated eggs are excreted into the environment through animal feces. Livestock and humans are infected by the ingestion of parasite eggs in contaminated food, water, soil, and by direct contact with infected dogs. The released embryo penetrates the intestinal mucosa and then develops into hydatid cyst, most frequently in the liver or lungs. However, other viscera and organs can also be affected.

At the present time, there is no best treatment choice for clinical management of human CE. However, the following four options are recommended by the WHOInformal Working Group on Echinococcosis: surgery, percutaneous treatment, anti-infective drug treatment and observation (watch and wait) for inactive and uncomplicated cysts (3). The availability of facilities, size, type, location of the cysts and the presence of symptoms are important elements in the adoption of therapeutic measures (1). Benzimidazole (BMZ) compounds such as albendazole (ABZ) and mebendazole are currently the most commonly used drugs, acting as an alternative to invasive surgery in patients with uncomplicated cysts, and in inoperable cases. Moreover, these drugs are indicated to prevent the recurrence of secondary cysts following surgery or the percutaneous treatment of CE cysts $(3,4)$. BMZ compounds destroy the parasite by the inhibition of tubulin polymerization, blockage of glucose absorption, and damaging of the organelles in the germinal layer (5). In clinical practice, however, about $20-40 \%$ of the patients do not respond adequately to these medications (6). Moreover, subsequent adverse effects including neutropenia, liver toxicity, and alopecia, often occur due to high daily doses 
and long-term administration of the drugs. Therefore, it is necessary to evaluate other BMZs alone or in combination with antiparasitic drugs for the effective treatment of CE.

Praziquantel (PZQ), a well-known anti-cestode drug (7), is less effective than ABZ for the treatment of human echinococcosis, and there is not enough supporting evidence to recommend the use of PZQ alone for the treatment of established or severe disseminated CE (8). However, the use of $\mathrm{PZQ}$, an isoquinolone derivative, in combination with $\mathrm{ABZ}$ was more effective than $\mathrm{ABZ}$ alone in the chemoprophylaxis of experimental hydatid cysts and in the treatment of intra-abdominal human CE (9-11). The rapid influx of calcium ions through the parasite's cell membrane resulting in strong contractions of the musculature and tegumental damage have been among the suggested anti-helminthic mechanisms of PZQ $(7,12)$.

Flubendazole (FLBZ) is another BMZ that has previously shown good efficacy against E. granulosus protoscoleces and metacestodes in in vitro and in vivo settings (13-15). FLBZ has also been more effective than ABZ against experimental secondary hydatidosis in mice (16). Despite the low plasma level of the drug, FLBZ have shown notable effects on the vitality of protoscoleces in the liver cyst of the treated sheep (17). Therefore, FLBZ appears to have potential as an alternative to $A B Z$ for the treatment of CE. As other BMZs, FLBZ impairs the glycolytic pathway and polymerization of tubulin subunits, resulting in reduction of glycogen stores (the most important form of energy storage), and finally kills the parasites. Given that FLBZ and PZQ affect the parasites from two different pathways, their simultaneous administration seems to be more effective. Accordingly, in this study we decided to evaluate the combined effect of FLBZ with PZQ against protoscoleces and microcysts of $E$. granulosus in vitro.

\section{Materials and Methods \\ Collection of Protoscoleces}

This experimental study was approved by research ethics committee of Zanjan University of Medical Sciences (ZUMS.REC.1394.197). Protoscoleces were collected aseptically from the liver and lungs of naturally infected sheep, slaughtered in an abattoir located in Zanjan province (18). They were washed several times with phosphate buffer saline solution (PBS), $\mathrm{pH} 7.2$, and the viability of protoscoleces was assessed by the eosin exclusion test (19). The intact morphological structure and the motility of flame cells were used to confirm the viability of protoscoleces. Only those batches with over $95 \%$ viability were used for the experiment.

\section{In vitro Culture and Treatment of Protoscoleces}

The washed protoscoleces were incubated $(1800 / \mathrm{mL})$ in $\mathrm{RPMI}_{1640}$ medium (Sigma-Aldrich, USA) containing $4 \mathrm{mg} / \mathrm{mL}$ of HEPES, $2 \mathrm{mg} / \mathrm{mL}$ of glutamine, $4 \mathrm{mg} / \mathrm{mL}$ of D-glucose, $100 \mathrm{IU} / \mathrm{mL}$ of penicillin and $100 \mu \mathrm{g} / \mathrm{mL}$ of streptomycin, supplemented with $10 \%$ fetal calf serum (FCS) at $37^{\circ} \mathrm{C}$ in $5 \% \mathrm{CO}_{2}(20)$.
Stock solutions of $0.1 \mathrm{mg} / \mathrm{mL}$ of FLBZ and PZQ (Sigma-Aldrich, USA) were prepared in dimethyl sulphoxide (DMSO), separately. The treatment of protoscoleces was initiated with final concentrations of $5 \mu \mathrm{g} / \mathrm{mL}$ FLBZ, $1 \mu \mathrm{g} / \mathrm{mL}$ PZQ and a combination of 5 $\mu \mathrm{g} / \mathrm{mL}$ FLBZ $+1 \mu \mathrm{g} / \mathrm{mL}$ PZQ. A culture treated with $\operatorname{DMSO}(1 \% v / v)$ and another without any treatment were used as control groups. The viability of protoscoleces was monitored for five consecutive days, and then until day 17 every other day, by microscopic observation and eosin exclusion test. The experiment was repeated three times and drug efficacies were presented as the mean value of the protoscoleces' viability rate. The significance of the differences between groups was determined by ANOVA using SPSS 16 (Inc. Chicago, IL, USA), and a P-value of less than 0.05 was considered statistically significant.

\section{In vitro Development and Treatment of Metacestodes}

For the development of microcysts, protoscoleces were cultured for 40 to 50 days in $\mathrm{RPMI}_{1640}$, in the same conditions for the in vitro culture of protoscoleces, except that the culture medium was renewed every 3-4 days $(21,22)$. Protoscoleces that had undergone stage conversion to microcysts (1-4 mm diameter) after 40-50 days of culture were selected and randomly allocated into three treatment and two control groups, with approximately 10 microcysts placed in $10 \mathrm{~mL}$ of culture medium. The drugs were added to the cultures with final concentrations of $5 \mu \mathrm{g} / \mathrm{mL}$ FLBZ, $1 \mu \mathrm{g} / \mathrm{mL}$ PZQ, and a combination of $5 \mu \mathrm{g} / \mathrm{mL}$ FLBZ $+1 \mu \mathrm{g} / \mathrm{mL}$ PZQ. Moreover, a culture treated with DMSO $(1 \% v / v)$ and another without any treatment were used as control groups. The degenerative changes of microcysts were monitored under an inverted light microscope (Motic $®$, AE31, Spain), every day for 21 days. The loss of cyst turgidity, and/or collapses of microcysts were used as criteria for microcyst degeneration, and as a result of drug efficacy (23).

Samples of microcysts were selected from each experiment, 21 days after treatment, and were processed for transmission electron microscopy (TEM) as described by Elissondo et al. with some modifycations (14). Briefly, the samples were fixed by $2.5 \%$ glutaraldehyde in PBS for $1 \mathrm{~h}$ at room temperature, washed 3 times with PBS, then post-fixed in $1 \% \mathrm{OsO}_{4}$ in PBS, followed by extensive washing with distilled water. The specimens were dehydrated in a graded series of acetone, and subsequently embedded in resin. Polymerization of the resin was carried out at $60^{\circ} \mathrm{C}$ for $48 \mathrm{~h}$. Ultrathin sections of $30-50 \mathrm{~nm}$ were made by LEICA ultramicrotome, stained with uranyl acetate saturated solution and lead citrate (30 minutes each levels), and observed using a transmission electron microscope (EM, LEO 906, Zeiss, Germany) at $80 \mathrm{kv}$. Degenerative ultra-structural changes including the detachment of the germinal layer from the outer laminated layer, the loss of microtriches, presence of numerous vacuoles and lipid droplets in the germinal layer as a result of drug efficacy were assessed (22). 


\section{Results}

\section{Treatment of Protoscoleces}

All the treated protoscoleces with the combination of $5 \mu \mathrm{g} / \mathrm{mL}$ FLBZ and $1 \mu \mathrm{g} / \mathrm{mL}$ PZQ were killed in 9 days post-treatment. Meanwhile, in the cultures that were treated independently with $5 \mu \mathrm{g} / \mathrm{mL}$ FLBZ or $1 \mu \mathrm{g} / \mathrm{mL}$ PZQ, up to $21.7 \pm 9.5 \%$ and $30.8 \pm 5.3 \%$ of protoscoleces were still alive, respectively (Table 1) $(P<0.05)$. The death of all protoscoleces only occurred 13- and 15days post-treatment with FLBZ and PZQ, respectively. In the control groups, more than $62 \%$ of protoscoleces were still alive on day 17 post-treatment. The drugs' protoscolicidal trends are presented in Figure 1.

Table 1. Viability of $E$. granulosus protoscoleces (percent; mean \pm SE) treated with the flubendazole (FLBZ, $5 \mu \mathrm{gg} / \mathrm{mL}) \mathrm{and}$ praziquantel (PZQ, $1 \mu \mathrm{g} / \mathrm{mL}$ ). Dimethylsolphoxide (DMSO, 1\% v/v).

\begin{tabular}{lcccccccccccc} 
Experiment & \multicolumn{7}{c}{ Treatment (day) } \\
\multicolumn{1}{c}{ Groups } & 1 & 2 & 3 & 4 & 5 & 7 & 9 & 11 & 13 & 15 \\
FLBZ & $92.9 \pm 8.0$ & $80.1 \pm 7.5$ & $72.0 \pm 7.1$ & $66.9 \pm 3.0$ & $53.8 \pm 8.5$ & $40.6 \pm 3.8$ & $21.7 \pm 9.5$ & $7.3 \pm 6.6$ & 0 & 0 \\
PZQ & $94.5 \pm 4.1$ & $86.0 \pm 8.3$ & $77.1 \pm 9.8$ & $66.5 \pm 3.4$ & $57.3 \pm 5.3$ & $46.2 \pm 2.6$ & $30.8 \pm 5.3$ & $15.4 \pm 1.2$ & $2.3 \pm 4.2$ & 0 & 0 \\
FLBZ+PZQ & $90.7 \pm 7.0$ & $76.5 \pm 4.1$ & $66.2 \pm 2.2$ & $53.7 \pm 2.3$ & $35.8 \pm 7.3$ & $16.3 \pm 5.1$ & 0 & 0 & 0 & 0 \\
DMSO & $93.7 \pm 8.0$ & $91.7 \pm 3.0$ & $87.5 \pm 6.0$ & $84.9 \pm 4.1$ & $83.5 \pm 7.1$ & $78.9 \pm 6.1$ & $76.3 \pm 6.2$ & $67.7 \pm 3.1$ & $69.1 \pm 5.0$ & $65.5 \pm 5.3$ & $62.1 \pm 7.3$ \\
no treatment & $95.5 \pm 8.1$ & $95.0 \pm 6.1$ & $93.3 \pm 2.2$ & $90.1 \pm 2.2$ & $89.5 \pm 2.0$ & $86.1 \pm 1.2$ & $86.6 \pm 1.2$ & $80.7 \pm 1.0$ & $76.1 \pm 6.1$ & $72.0 \pm 5.2$ & $69.8 \pm 6.1$ \\
\hline
\end{tabular}

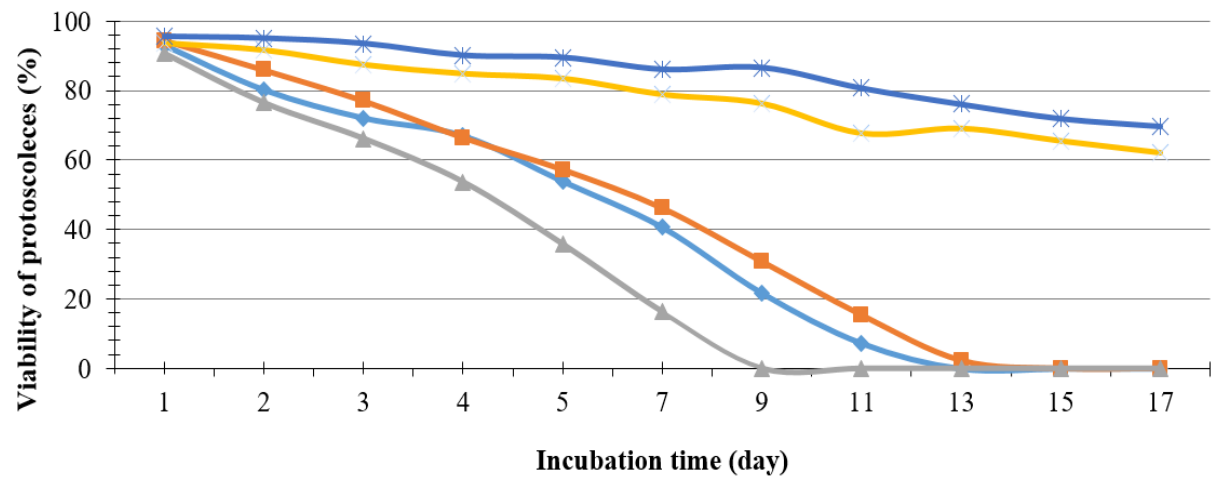

$\leadsto$ FLBZ $(5 \mu \mathrm{g} / \mathrm{ml}) \rightarrow$ PZQ $(1 \mu \mathrm{g} / \mathrm{ml}) \rightarrow$ FLBZ + PZQ $\longrightarrow$ DMSO (control) $\rightarrow$ no treatment

Figure 1. Viability of E. granulosus protoscoleces treated with the flubendazole (FLBZ, $5 \mu \mathrm{g} / \mathrm{mL}$ ) and praziquantel (PZQ, 1 $\mu \mathrm{g} / \mathrm{mL}$ ). Dimethylsolphoxide (DMSO, $1 \% v / v$ ).
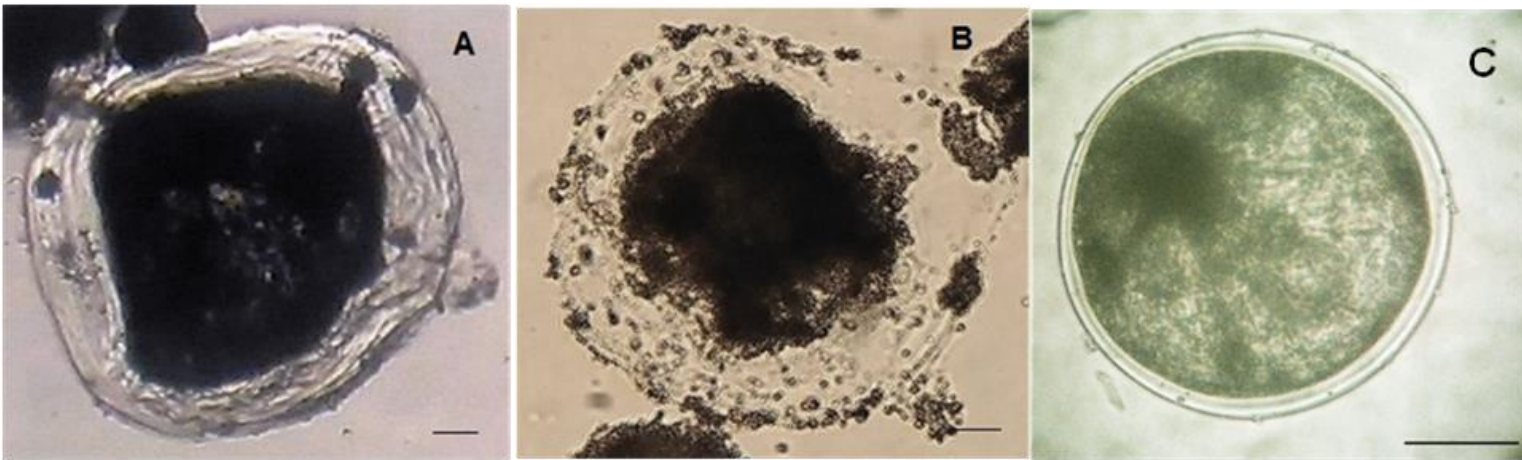

Figure 2. Light microscopy of E granulosus microcyst treated with $5 \mu \mathrm{g} / \mathrm{mL}$ FLBZ $+1 \mu \mathrm{g} / \mathrm{mL}$ PZQ. (A) microcyst, four days posttreatment, (B) The same microcyst 17 days post-treatment, and (C) intact microcyst from control. Scale bars: $100 \mu \mathrm{m}$. 


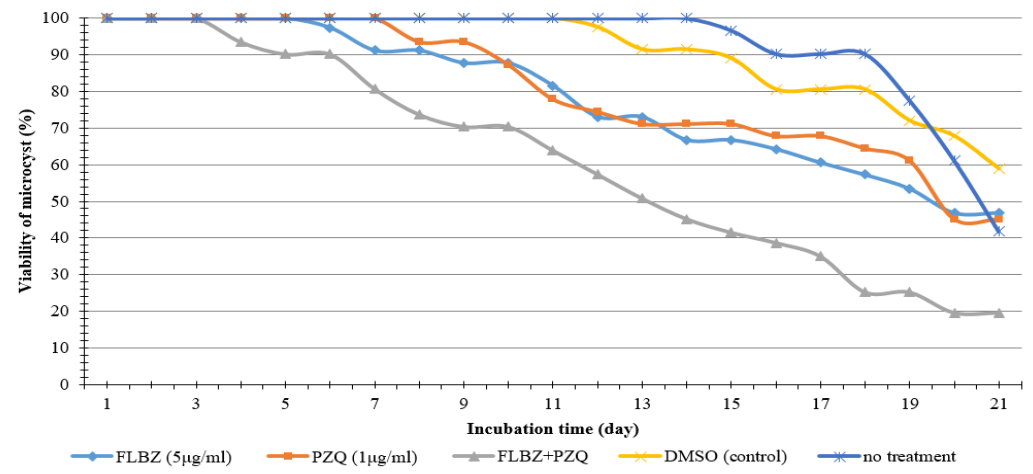

Figure 3.Viability of E. granulosus microcysts treated with the flubendazole (FLBZ, $5 \mu \mathrm{g} / \mathrm{mL}$ ) and praziquantel (PZQ, $1 \mu \mathrm{g} / \mathrm{mL})$. Dimethylsolphoxide (DMSO, $1 \% \mathrm{v} / \mathrm{v}$ ).

\section{Treatment of Microcysts}

The signs of microcyst degeneration were visible microscopically in the form of loss of cyst turgidity, collapse and formation of a densely packed aggregate inside the cysts (Figure 2). The loss of cyst turgidity and other degenerative changes in microcysts treated with $5 \mu \mathrm{g} / \mathrm{mL}$ FLBZ $+1 \mu \mathrm{g} / \mathrm{mL}$ PZQ were observed on day 4 post-treatment, while these alterations in microcysts treated with FLBZ or PZQ alone occurred two and four days later, respectively. Approximately, more than $70 \%$ of microcysts treated with FLBZ or PZQ alone were viable (intact) on day
13 post-treatment, whereas only $50 \%$ of microcysts treated with both FLBZ and PZQ were viable on the same day $(P<0.05)$ (Figure 3$)$. The rate of viable microcysts in DMSO and no treatment groups were $91 \%$ and $100 \%$, respectively. These findings were confirmed through ultrastructural observation by TEM (Figure 4). The microcysts treated with both FLBZ and PZQ showed microtriches atrophy, the presence of numerous vacuoles, lipid droplets and dense mitochondria in germinal layer, but the control microcysts exhibited the typical appearance of $E$. granulosus metacestodes structures.
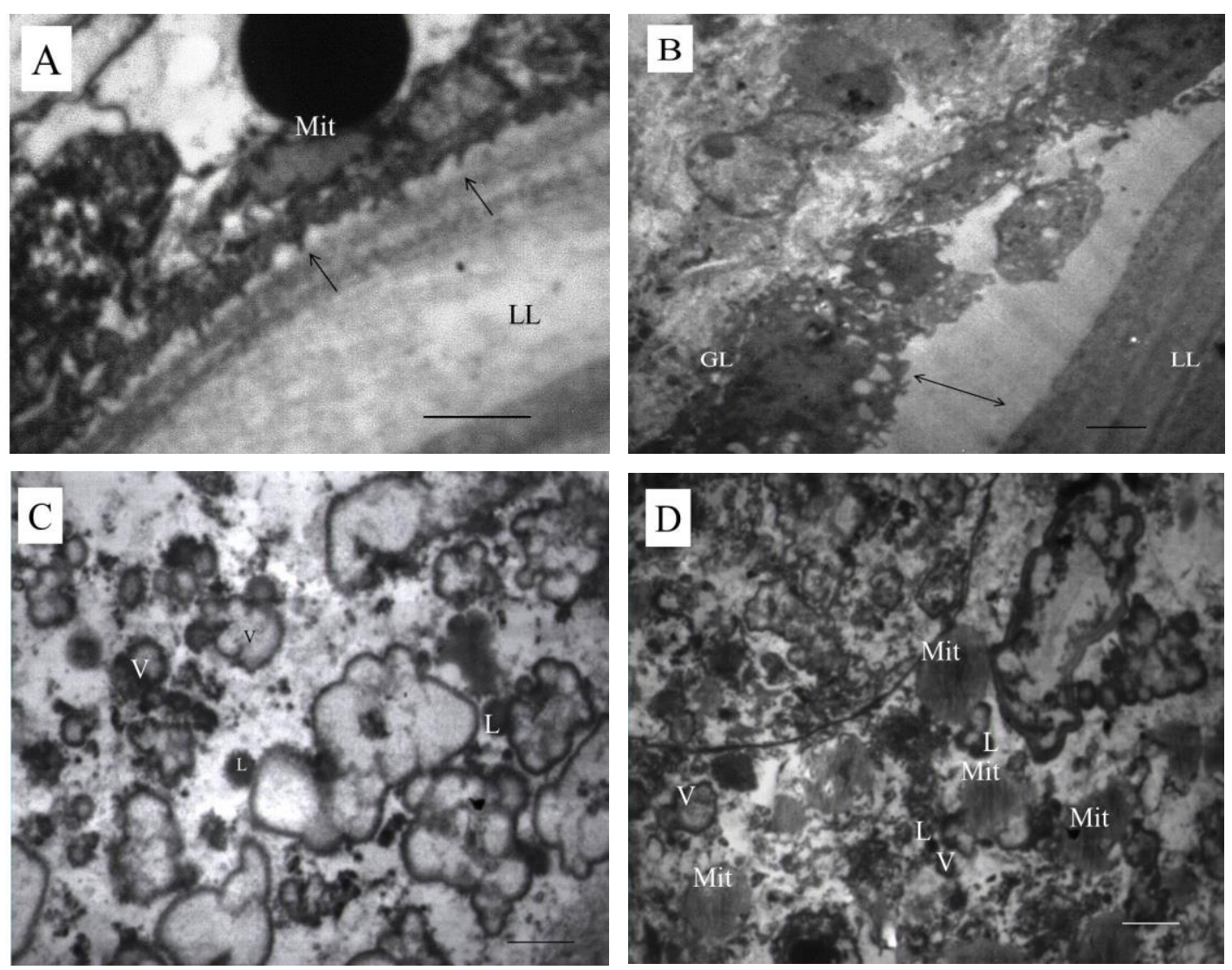

Figure 4. Transmission electron microscopy of E. granulosus microcysts: (A) Normal ultra-structure of microcysts in control group (× 2784), arrows show normal microtriches. (B-D) Microcysts incubated with FLBZ $(5 \mu \mathrm{g} / \mathrm{mL})+\mathrm{PZQ}(1 \mu \mathrm{g} / \mathrm{mL})$. (B) Arrow shows germinal layer detachment and loss of microtriches $(\times 1000)$. (C) Presence of numerous vacuoles and lipid droplets in distal cytoplasm $(\times 2784)$, and (D) Electron-dense and rounded mitochondria presence in the GL of microcysts $(\times 1670)$. Scale bars: $10 \mu \mathrm{m}$. GL) germinal layer; LL) laminated layer; Mit) mitochondria; L) lipid droplets, and V) vacuole 


\section{Discussion}

The efficacy of FLBZ against E. granulosus protoscoleces and microcysts has previously been studied either alone $(13,14)$ or in combination with ivermectin (15) in a few in vitro or in vivo investigations. However, in the present study, the combined effect of FLBZ with PZQ was studied on the protoscoleces and microcysts for the first time. Results showed that all of the protoscoleces treated with FLBZ and PZQ were killed in nine days. While the cultures that were treated either with FLBZ or PZQ, 21.7 $\pm 9.5 \%$ and $30.8 \pm 5.3 \%$ of protoscoleces were still viable, and the death of all protoscoleces only occurred 13 and 15 days post-treatment, respectively. Moreover, degenerative changes in microcysts such as the loss of cyst turgidity, collapse and formation of a densely packed aggregates inside the microcysts treated with both FLBZ and PZQ were observed to occur faster (four days post-treatment) than those treated with FLBZ or PZQ alone (six- and eight-days post-treatment, respectively).

Studies showed that the protoscoleces cultured with FLBZ were killed considerably faster than those cultured with ABZ or ABZ-sulphoxide $(13,24)$, so that after 25 days of exposure to $5 \mu \mathrm{g} / \mathrm{mL}$ FLBZ, the viability of protoscoleces was about $14 \%$ (13), but the reported viability of protoscoleces for $\mathrm{ABZ}$ or $\mathrm{ABZ}$ sulphoxide was approximately $50 \%$ after 24 days of culture (24). However, in the present study all protoscoleces were dead 13 days after incubation with $5 \mu \mathrm{g} / \mathrm{mL}$ FLBZ.

The survival of E. granulosus protoscoleces after exposure to FLBZ, ivermectin (IVM) and their combination was also studied. The maximum protoscolicidal effects were found to be with the combination of $10 \mu \mathrm{g} / \mathrm{mL}$ of FLBZ $+1 \mu \mathrm{g} / \mathrm{mL}$ of IVM, in which the viability of protoscoleces was reduced to less than 5\% after 24 days of treatment (15). But, in our study all of the protoscoleces that were incubated with FLBZ+PZQ were dead after nine days, showing the better protoscolicidal efficacy of $\mathrm{FLBZ}+\mathrm{PZQ}$ in comparison to FLBZ+IVM.

There are a few studies available concerning the efficacy of PZQ against E. granulosus protoscoleces. Thompson et al. previously showed that $10 \mu \mathrm{g} / \mathrm{mL}$ of PZQ caused the death of protoscoleces within 12 to 15 days (25), while in the present study the same protoscolicidal efficacy was achieved with a lower concentration of PZQ $(1 \mu \mathrm{g} / \mathrm{mL})$ in the same period, which could be due to the drug solvent, incubation conditions or even the parasite's strain. For instance, they used CMRL-1066 as a culture medium and Hank's buffered salt solution for dissolving PZQ, while in the present study, $\mathrm{RPMI}_{1640}$ was used as culture medium and PZQ was dissolved in DMSO. According to some evidence, increasing PZQ dissolution could lead to better transdermal delivery of the drug in the animal model (26).
Although PZQ is less effective than ABZ for the treatment of $\mathrm{CE}$ (8), the combination of PZQ with some drugs has already been shown to have better antiparasitic effects. PZQ in combination with ABZ had more prophylactic efficacy for the treatment of experimental hydatidosis $(9,12)$. In a study, a combination therapy of PZQ and ABZ killed approximately $50 \%$ of protoscoleces after 15 days of treatment (27), while in our experiment, it took only four days to kill at least $50 \%$ of the protoscoleces which shows the better synergistic effect of PZQ with FLBZ (Table 1).

In the present study, the appearance of collapsed microcysts was on the sixth and eighth days of treatment with $5 \mu \mathrm{g} / \mathrm{mL}$ of FLBZ and $1 \mu \mathrm{g} / \mathrm{mL}$ of PZQ, respectively. Microcysts incubated with a combination of these two drugs collapsed earlier, on the fourth day of treatment (Figure 3). In line with this finding, collapsed cysts were seen on the sixth day following treatment with FLBZ alone (14), but when treated with combination of FLBZ $(10 \mu \mathrm{g} / \mathrm{mL})$ and IVM $(1 \mu \mathrm{g} / \mathrm{mL})$, the cysts collapsed on the second day of treatment, earlier than FLBZ monotherapy (15).

The ultra-structural findings of microcysts treated with the FLBZ and/or PZQ are consistent with those reported by Casado $(23)$, Elissondo $(13,14)$ and Walker (21). An increase of vacuolization in the distal cytoplasm, the presence of lipid droplets, and the loss of microtriches were observed. Since the increased amount of vacuolation occurs in protoscoleces and metacestodes exposed to several drugs with different modes of action, Perez-Serrano suggested that it could reflect general tissue stress as a consequence of an indirect effect of the drugs (24).

Loss or atrophy of microtriches in the germinal layer of microcysts was a similar finding to those results from other benzimidazoles $(13,28)$. Benzimidazoles act on parasite tubulin and inhibits the formation of microtubules (29). Alteration in the tegumental microtriches of protoscoleces probably interferes with parasite nutrition, since microtriches are directly associated with nutrient absorption and their alteration contributes to loss of protoscolex viability (24).

\section{Conclusion}

Combination of FLBZ with PZQ is more effective than each drug alone against E. granulosus protoscoleces and microcysts, but further in vivo studies are required to evaluate the potential of this drug combination against human hydatidosis.

\section{Acknowledgments}

The authors acknowledge the Research Vicechancellor of Zanjan University of Medical Sciences for financial support of this work (A-12-95-9). The authors have no conflict of interest to declare. 


\section{Conflict of Interest}

Authors declare no conflict of interests.

\section{References}

1. Agudelo Higuita NI, Brunetti E, McCloskey C. Cystic echinococcosis. J Clin Microbiol. 2016; 54(3): 518-23. [DOI:10.1128/JCM.02420-15] [PMID] [PMCID]

2. World Health Organisation. Echinococcosis. World Health Organisation, Geneva, Switzerland. http://www.who.int /mediacentre/factsheets/fs377/en Accessed on 12 Feb 2018.

3. Brunetti E, Kern P, Vuitton DA, Writing Panel for the WHOIWGE. Expert consensus for the diagnosis and treatment of cystic and alveolar echinococcosis in humans. Acta trop. 2010; $114(1)$ : $1-16$ [DOI:10.1016/j.actatropica.2009.11.001] [PMID]

4. McManus DP, Gray DJ, Zhang W, Yang Y. Diagnosis, treatment, and management of echinococcosis. BMJ. 2012; 344: e3866. [DOI:10.1136/bmj.e3866] [PMID]

5. Teggi A, Lastilla MG, De Rosa F. Therapy of Human Hydatid-Disease with Mebendazole and Albendazole. Antimicrob Agents Chemother. 1993; 37(8): 1679-84. doi:10.1128/aac.37.8.1679 [DOI:10.1128/AAC.37.8.1679] [PMID] [PMCID]

6. Moro P, Schantz PM. Echinococcosis: a review. Int J Infect Dis. 2009; 13(2): 125-33. [DOI:10.1016/j.ijid.2008.03.037] [PMID]

7. Chai JY. Praziquantel treatment in trematode and cestode infections: an update. Infect Chemother. 2013; 45(1): 32-43 [DOI:10.3947/ic.2013.45.1.32] [PMID] [PMCID]

8. Bygott JM, Chiodini PL. Praziquantel: neglected drug? Ineffective treatment? Or therapeutic choice in cystic hydatid disease? Acta Trop. 2009; 111(2): 95-101. [DOI:10.1016/i.actatropica.2009.04.006] [PMID]

9. Casado N, Urrea-Paris MA, Moreno MJ, RodriguezCaabeiro F. Combined praziquantel and albendazole chemoprophylaxis in experimental hydatidosis. Parasitol Res. 2001; 87(9): 787-9. [DOI:10.1007/s004360100443] [PMID]

10. Mohamed AE, Yasawy MI, Al Karawi MA. Combined albendazole and praziquantel versus albendazole alone in the treatment of hydatid disease. Hepato-Gastroenterol. 1998; 45(23): 1690-4.

11. Nazligul Y, Kucukazman M, Akbulut S. Role of chemotherapeutic agents in the management of cystic echinococcosis. Int Surg. 2015; 100(1): 112-4. [DOI:10.9738/INTSURG-D-14-00068.1] [PMID][PMCID]

12. Urrea-Paris MA, Casado N, Moreno MJ, RodriguezCaabeiro F. Chemoprophylactic praziquantel treatment in experimental hydatidosis. Parasitol Res. 2001; 87(6): 510-2. [DOI:10.1007/s004360100390]
13. Elissondo M, Dopchiz M, Ceballos L, et al. In vitro effects of flubendazole on Echinococcus granulosus protoscoleces. Parasitol Res. 2006; 98(4): 317-23. [DOI:10.1007/s00436005-0026-6] [PMID]

14. Elissondo M, Ceballos L, Dopchiz M, et al. In vitro and in vivo effects of flubendazole on Echinococcus granulosus metacestodes. Parasitol Res. 2007; 100(5): 1003-9. [DOI:10.1007/s00436-006-0381-y] [PMID]

15. Elissondo MC, Ceballos L, Alvarez L, Bruni SS, Lanusse C, Denegri G. Flubendazole and ivermectin in vitro combination therapy produces a marked effect on Echinococcus granulosus protoscoleces and metacestodes. Parasitol Res. 2009; 105(3): 835-42. [DOI:10.1007/s00436009-1469-y] [PMID]

16. Ceballos L, Elissondo C, Bruni SS, Denegri G, Lanusse C, Alvarez L. Comparative performances of flubendazole and albendazole in cystic echinococcosis: ex vivo activity, plasma/cyst disposition, and efficacy in infected mice. Antimicrob agents chemother. 2011; 55(12): 5861-7. doi:10.1128/AAC.05105-11 [DOI:10.1128/AAC.05105-11] [PMID] [PMCID]

17. Ceballos L, Virkel G, Elissondo C, et al. A pharmacologybased comparison of the activity of albendazole and flubendazole against Echinococcus granulosus metacestode in sheep. Acta trop. 2013; 127(3): 216-25. [DOI:10.1016/j.actatropica.2013.05.004] [PMID]

18. Haniloo A, Ghasemi F, Shikhi Ak, Ghavami MB. Immunoregulatory cytokine (TGF-B And IL-10) responses in mice inoculated with protoscoleces and major hydatid fluid antigens of cystic echinococcosis. Iranian J Parasitol. 2008; 3(3): 18-23.

19. Haniloo A, Najafi F, Fazaeli A, Nourian A. Comparison and evaluation of Echinococcus granulosus protoscoleces excretory/secretory proteins in PBS complemented with glucose, DMEM and RPMI culture media. J Zanjan Univ Med Sci. 2011; 19 (74): 44-53.

20. Liu CS, Zhang HB, Yin JH, Jiang B, Han XM. Echinococcus granulosus: Suitable in vitro protoscolices culture density. Biomed Environ Sci. 2013; 26(11): 912-5

21. Walker M, Rossignol JF, Torgerson P, Hemphill A. In vitro effects of nitazoxanide on Echinococcus granulosus protoscoleces and metacestodes. J Antimicrob Chemother. 2004; 54(3): 609-16. [DOI:10.1093/jac/dkh386] [PMID]

22. Naguleswaran A, Spicher M, Vonlaufen N, et al. In vitro metacestodicidal activities of genistein and other isoflavones against Echinococcus multilocularis and Echinococcus granulosus. Antimicrob Agents Chemother. 2006; 50(11): 3770-8. [DOI:10.1128/AAC.00578-06] [PMID] [PMCID]

23. Casado N, Perez-Serrano J, Denegri G, Rodriguez-Caabeiro F. Development of a chemotherapeutic model for the in vitro screening of drugs against Echinococcus granulosus cysts: The effects of an albendazole sulphoxide combination. Int $\mathrm{J}$ Parasitol. 1996; 26(1): 59-65. [DOI:10.1016/00207519(95)00095-X] 
24. Perez-Serrano J, Casado N, Denegri G, Rodriguez-Caabeiro F. The effects of albendazole and albendazole sulfoxide combination-therapy on Echinococcus granulosus in vitro. Int J Parasitol. 1994; 24(2): 219-24. [DOI:10.1016/00207519(94)90029-9]

25. Thompson RC, Reynoldson JA, Riddler CR. Praziquantel adversely affects protoscoleces of Echinococcus granulosus in vitro. J Helminthol. 1986; 60(4): 279-86. [DOI:10.1017/S0022149X00008488]

26. Zheng XS, Duan CZ, Xiao ZD, Yao BA. Transdermal delivery of praziquantel: effects of solvents on permeation across rabbit skin. Biol Pharm Bull. 2008; 31(5): 1045-8. [DOI:10.1248/bpb.31.1045] [PMID]
27. Urrea-Paris MA, Moreno MJ, Casado N, RodriguezCaabeiro F. In vitro effect of praziquantel and albendazole combination therapy on the larval stage of Echinococcus granulosus. Parasitol Res. 2000; 86(12): 957-64. [DOI:10.1007/PL00008526]

28. Perez-Serrano J, Denegri G, Casado N, Rodriguez-Caabeiro F. In vivo effect of oral albendazole and albendazole sulphoxide on development of secondary echinococcosis in mice. Int J Parasitol. 1997; 27(11): 1341-5. [DOI:10.1016/S0020-7519(97)00105-7]

29. Lacey E. The role of the cytoskeletal protein, tubulin, in the mode of action and mechanism of drug resistance to benzimidazoles. Int J Parasitol. 1988; 18(7): 885-936. [DOI:10.1016/0020-7519(88)90175-0]

\section{How to Cite This Article:}

Pezeshki A, Lorestani S, Torabi N, Haniloo A. Combined Effect of Flubendazole and Praziquantel on Echinococcus granulosus Protoscoleces and Microcysts. J Adv Med Biomed Res. 2018; 26 (118):34-40

\section{Download citation:

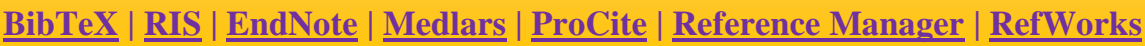

\section{Send citation to:}

Mendeley 2 Zotero (1) RefWorks RefWorks 